\title{
IDENTIFICACIÓN DE LA RAZA CRIOLLO CAQUETEÑO MEDIANTE EL ESTUDIO DE LAS CARACTERÍSTICAS FANERÓPTICAS
}

\author{
Identification of the creole race caqueteño through the study of the \\ fanerópticas characteristics
}

\section{Artículo de Investigación}

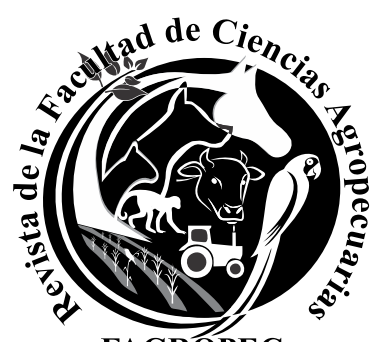

FAGROPEC

Recibido 21 de enero de 2019. Aceptado 15 de marzo de 2019.

${ }^{1}$ Médico Veterinario UDCA, Mg Docente de la Facultad de Ciencias Agropecuarias de la Universidad de la Amazonía, Grupo de Investigación en Fauna Silvestre.

(iD) https://orcid.org/0000-0001-5773-2729

${ }^{2}$ Zootecnista Universidad Nacional de Colombia. $\mathrm{Mg}$ Animal Nutrition University of Florida, $\mathrm{PhD}$ Animal Production University of Reading, Docente Facultad de Ciencias Básicas de la Universidad de la Amazonía, Grupo de Investigación GIPSA

(iD) https://orcid.org/0000-0003-2169-8017

${ }^{3}$ Zootecnista Universidad de Nariño, $\mathrm{Mg}$ Agroforestería, Docente de la Facultad de Ciencias Agropecuarias de la Universidad de la Amazonía, Grupo de Investigación GISAPA

https://orcid.org/0000-0003-3235-886X

${ }^{4}$ Médico Veterinario Zootecnista Universidad de la Amazonía, Mg Docente de la Facultad de Ciencias Agropecuarias de la Universidad de la Amazonía, Grupo de Investigación en Fauna Silvestre.

https://orcid.org/0000-0002-0246-6125

*Autor para correspondencia: b.patiño@udla.edu.co
Patiño Quiroz Beatriz Elena ${ }^{1 *}$, Velásquez Restrepo Jaime Enrique ${ }^{2}$, Ocaña Martínez Hernán Eduardo ${ }^{3}$, Baldrich Romero Nicolás $\mathbf{E}^{4}$

\section{RESUMEN}

Los estudios fanerópticos permiten identificar, describir y diferenciar las particularidades externas de los animales que serán seleccionados para mejorar los procesos de consolidación de las razas. El ganado Criollo Caqueteño ha sobrevivido gracias a su rusticidad y adaptación como resultado de la selección natural a las condiciones del piedemonte amazónico colombiano, convirtiéndose en una alternativa para productores de la región. En la presente investigación se identifican los caracteres fanerópticos de individuos de la raza. Se observaron 98 ejemplares (34 machos y 64 hembras) en tres predios del departamento del Caquetá y se analizaron nueve características. Producto de esta investigación se identificaron dos colores, rojo ( $79 \%$ ) y bayo (21\%); con pelo corto y fino; el $84 \%$ de los animales presentó papada discontinua y el color de las mucosas se agrupó en sonrosadas con 46 individuos y oscurecidas en 52 individuos. No se encontró dimorfismo sexual. Dadas las características fenotípicas identificadas en el actual estudio, el bovino Criollo Caqueteño se proyecta como una raza con las condiciones adecuadas para dar rusticidad y resistencia a los cruces en la ganadería, sin embargo, el bajo número de la población prende las alarmas sobre la necesidad de plantear programas para su conservación y rescate.

Palabras claves:

Ganado; Criollo; Raza; Preservación; Estándar Racial.

\section{ABSTRACT}

The phaneroptic studies allow to identify, describe and differentiate the external particularities of the animals that will be selected to improve the consolidation processes of the breeds. The Caqueteño Creole cattle has survived thanks to its rusticity and adaptation as a result of natural selection to the conditions of the Colombian Amazonian piedmont, becoming an alternative for producers in the region. In the present investigation, the phaneroptic characters of individuals of the breed are identified. Ninetyeight individuals (34 males and 64 females) were studied in three farms in the Department of Caquetá and nine characteristics were analyzed. As a result of this investigation, two colors were identified, red (79\%) and bay (21\%); with short fine hair; $84 \%$ of the animals presented discontinuous 


\section{Como citar:}

PATIÑO-QUIROZ, B.E. et al. Identificación de la raza criollo caqueteño mediante el estudio de las características fanerópticas. En: Revista Facultad Ciencias Agropecuarias FAGROPEC. Universidad de la Amazonia, Florencia - Caquetá. Volumen 11 enero-junio, 2019. Pp. 23-32. ISSN-Revista en Línea: 2539$178 \mathrm{X}$ dewlap and the color of the mucosa was grouped into rosy with 46 individuals and darkened in 52 individuals. No sexual dimorphism was found. Given the phenotypic characteristics identified in the current study, the Caqueteño Creole bovine is projected as a race with the right conditions to give rusticity and resistance to the crossings in livestock, however, the low number of the population triggers the alarms on the need to raise programs for their conservation and rescue.

Key words:

Creole; Cattle; Breed; Preservation; Racial Standard.

\section{INTRODUCCIÓN}

Los recursos genéticos agrupados bajo la concepción de razas bovinas se conciben como el resultado de la intervención humana, mediante selección y cruzamiento a través de miles de años. No fue sino hasta el siglo XVIII cuando en Europa las diferencias entre el ganado adquirieron nombre y comenzó a utilizarse el término "Raza" (Eureca, 2010). Según el sistema de información sobre razas de animales domésticos (DAD-IS en inglés) para el 2014 se encontraban registradas 1099 razas bovinas, y en el 2004 se estimaba una población mundial de 1.339 millones de cabezas FAO, 2015). De estas, para el 2016 Colombia contaba con el 1,7\% del total; con una diversidad de razas y cruces aptos para diversos sistemas de producción y para ciertos pisos térmicos.

La FAO (2012a) define las razas indígenas/autóctonas/nativas como aquellas "Procedentes de, adaptado a y utilizada en una región geográfica determinada, forman un subconjunto de las Razas adaptadas localmente". Los animales criollos, entre muchas otras bondades, tienen una amplia adaptación climática, reproductiva, nutricional, sanitaria, capacidad de aprovechamiento de recursos naturales y de supervivencia con limitados recursos alimenticios disponibles, al igual que responden bien a los sistemas de ceba intensiva (Barrera, 2006; Sierra, 2001). Estos rasgos generan en los investigadores nacionales e internacionales interés, por lo que se reportan estudios de alto impacto sobre estos individuos y paralelismos con otras poblaciones españolas (Barba, 2005)

Las razas de bovinos criollos colombianos, son descendientes del ganado traído por los españoles en el segundo viaje de Colón en 1493 a la isla La Española, hoy República Dominicana y Haití (Primo, 1992). Estos animales se distribuyeron junto con los pobladores españoles y fueron asentándose por el continente americano en diferentes latitudes, en algunos casos, con muy poca intervención humana, lo que ocasionó situaciones particulares como en Colombia, donde se desarrollaron ocho razas nativas con poblaciones que hoy se encuentran seriamente disminuidas, y entre las que se cuentan las siguientes con su respectivo número de ejemplares: Romosinuano (2.014) y Costeño con Cuernos (416) en la Costa Atlántica; Blanco Orejinegro (2.886) y Chino Santandereano (368) en la zona montañosa; Hartón del Valle (HV) (5.120) en el Valle del río Cauca, Casanareño (421) y San Martinero (3.166) en la Orinoquía y el Criollo Caqueteño $(<200)$ en el Amazonia (Gutiérrez et al, 2003; Alderson, 1974; Ortiz, 1997).

El bovino de raza Criollo Caqueteño (GCC) enmarca la historia de los colonizadores a cargo de los frailes franciscanos, los cuales emprendieron la colonización del territorio de la Amazonia Caqueteña. Las condiciones ambientales de valles y planicies convertidas a praderas para la siembra 
de gramíneas en zonas antiguamente boscosas dieron condiciones adecuadas para su proliferación, pero dentro de un proceso de adaptación al entorno durante casi 5 siglos. Bajo esas condiciones, la raza desarrolló características de rusticidad y resistencia (Barrera (2006). Este autor caracterizó la población a partir de 80 ejemplares para lo cual utilizó 14 marcadores moleculares tipo microsatélite, y reporta que estos animales son Bos Taurus, con una población genéticamente uniforme y separada de otras razas criollas. Este estudio también demostró que el GCC se relaciona con las razas BON y Romo.

Una de las herramientas más importantes en la selección de los especímenes es el conocimiento etnológico que abarca las características genéticas, físicas y comportamentales de los individuos (Buxadé, 1995). La faneróptia es definida por Sánchez y Sánchez (1986), como la condición visible; de la morfología externa aplicada a la etnología bovina, que estudia las estructuras perceptibles de base tegumentaria y de cobertura. La faneróptica abarca el estudio de la piel, como carácter étnico la dermis, dotación glandular, caracteres del pelo y de la lana (estructura), coloraciones, encornaduras, uñas, pezuñas, entre otros (Rodero, 2002). De acuerdo con Sañudo (2009), las investigaciones de índole faneróptica permiten describir, diferenciar e identificar las características externas de los animales que deberán ser seleccionadas para mantener y mejorar en los procesos de consolidación de las razas.

La raza de bovinos Criollo Caqueteño cuenta con un bajo remanente de hembras en capacidad reproductiva $(<150$ individuos), ubicando a esta población de ganado actualmente en la categoría "en peligro de extinción". Teniendo en cuenta los parámetros que para tal efecto ha establecido la FAO en 1990; la erosión genética que representaría la pérdida de este grupo racial, supondría una grave fragmentación y pérdida de los códigos biológicos desarrollados en la línea del acostumbramiento de este tipo de bovinos.

El presente trabajo se fundamenta en la necesidad de desarrollar evidencias científicas que permitan su adecuada identificación, uso y aprovechamiento, en correspondencia con los lineamientos de la FAO (2012b) que promueven el uso de razas o variedades productivas del sector agropecuario, naturalmente adaptada a las zonas en las que están siendo aprovechadas. Este documento presenta los resultados de un estudio de las características fanerópticas de la raza Criollo Caqueteño en la Amazonia colombiana.

\section{MATERIALES Y MÉTODOS}

Área del estudio: La investigación de tipo descriptiva se desarrolló en el departamento del Caquetá, en tres predios rurales con un estimado del $75 \%$ de la población de Bovinos de la Raza Criollo Caqueteño. Los predios se localizan geográficamente en la Amazonia colombiana; en un clima AF (Ecuatorial) según la clasificación de Köppen y dentro del ecosistema de bosque húmedo tropical (Holdridge, 1978). En estos predios se reporta una temperatura promedio entre $24,4^{\circ} \mathrm{C}$ y $26^{\circ} \mathrm{C}$ una pluviosidad anual que reporta desde $3793 \mathrm{~mm}$ hasta $3340 \mathrm{~m}$, brillo solar de 1707 horas/luz/año, humedad relativa $85,7 \%$ (IDEAM, 2009). Las observaciones se realizaron en las siguientes localidades:

1.Centro de Investigaciones Amazónicas CIMAZ- MACAGUAL "Cesar Augusto Estrada 
Gonzáles", de la Universidad de la Amazonia, ubicado a $20 \mathrm{~km}$ del casco urbano del municipio de Florencia; $1^{\circ} 37^{\prime} \mathrm{N}$ y $75^{\circ} 36^{\prime} \mathrm{W}$.

2.Explotación Villa Mery, ubicada aproximadamente a $5 \mathrm{~km}$ del casco urbano del municipio de Morelia, vía a Valparaíso; $1^{\circ} 48^{\prime} \mathrm{N}$ y $75^{\circ} 72^{\prime} \mathrm{W}$.

3.Finca el Pensil, ubicada en el km 2 sobre la vía Montañita - Florencia; 01²8'08.8” N y $075^{\circ} 27^{\prime} 31.8^{\prime \prime} \mathrm{W}$.

En total se utilizaron 98 bovinos distribuidos así: de las fincas El Pensil y Villa Mery se evaluaron 10 y 18 vacas, respectivamente, mientras que de Macagual se evaluaron 36 hembras, 31 machos y 3 toros.

Implicaciones éticas: Durante el periodo de muestreo todos los animales fueron tratados de acuerdo con la normatividad actual de bienestar animal, evitando cualquier evento que pudiera ocasionar estrés a los semovientes (Estrada, 2014). Esta investigación fue avalada por el comité de Ética Bioética y Bienestar Animal de la Universidad de la Amazonia medianteActa del 23 de abril de 2014. Donde se observó directamente el fenotipo de cada individuo y se determinaron los caracteres fanerópticos siguiendo algunos lineamientos de Sañudo (2009).

Análisis estadístico: Los resultados se analizaron en general mediante estadísticas descriptivas. Se aplicó una prueba de $x^{2}$ para determinar posibles asociaciones entre las características y el sexo. En algunas de las características con nombres combinados, donde el número de individuos era muy bajo o cero, los valores se sumaron a las características predominantes para realizar la prueba $x^{2}$; en otras, donde era evidente la dominancia de un carácter en particular y se presentaban valores muy bajos en otros, no se realizó la prueba y solo se presentan los porcentajes de estas. Se realizó un análisis de correspondencia múltiple utilizando el paquete estadístico R (R-core team, 2018).

\section{RESULTADOS Y DISCUSIÓN}

Los rasgos fanerópticos de bovinos de la raza Criollo Caqueteño se presentan en porcentaje para machos y hembras, al igual que el valor de $x^{2}$, en la tabla 1 .

Los resultados de la Tabla 1 indican que, con excepción del color de las mucosas, no se encontró significancia $(\mathrm{P}<0,05)$ entre las características y el sexo, según la prueba $x^{2}$. Esto, en términos generales, indica que no hay un dimorfismo sexual en la faneroptia de la raza Criollo Caqueteño, corroborado con un análisis de correspondencia multiple (ACM) presentado en la figura 1 .

En la figura 1, el ACM muestra la mayoría de los animales hacia el centro del cuadrante indicando poca dispersión. De igual manera, las elípticas indican que no hay una diferencia significativa $(\mathrm{P}<0,05)$ entre machos $\mathrm{y}$ hembras para las

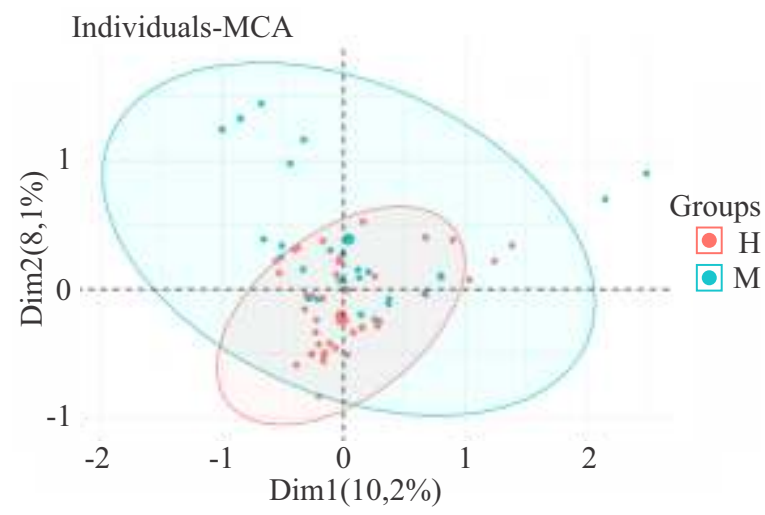

Figura 1. Análisis de Correspondencia Múltiple de variables fanerópticas de machos y hembras de ganado Criollo Caqueteño. 
Tabla 1. Características fanerópticas de ganado Criollo Caqueteño (\%)

\begin{tabular}{|c|c|c|c|c|}
\hline Carácter & Característica & Hembras & Machos & $x^{2}$ \\
\hline \multirow[t]{3}{*}{ Papada } & & & & 0,288 \\
\hline & Discontinua & 87,5 & 79,4 & \\
\hline & Continua & 12,5 & 20,6 & \\
\hline \multirow[t]{3}{*}{ Capa } & & & & 0,882 \\
\hline & Rojo & 78,1 & 79,4 & \\
\hline & Bayo & 21,9 & 20,6 & \\
\hline \multirow[t]{3}{*}{ Pliegue umbilical } & & & & 0,122 \\
\hline & Ausente & 68,8 & 52,9 & \\
\hline & Presente & 31,2 & 47,1 & \\
\hline \multirow[t]{3}{*}{ Color mucosas } & & & & $0,011^{*}$ \\
\hline & Sonrosadas & 56,2 & 29,4 & \\
\hline & Oscurecido-negra & 43,8 & 70,6 & \\
\hline \multirow[t]{3}{*}{ Manchas } & & & & 0,749 \\
\hline & Ausente & 85,9 & 88,2 & \\
\hline & Algunas manchas & 14,1 & 11,8 & \\
\hline \multirow[t]{4}{*}{ Borla } & & & & $\mathrm{ND} * *$ \\
\hline & Rojo & 93,8 & 82,3 & \\
\hline & Negro & 3,1 & 5,9 & \\
\hline & Amarillo & 3,1 & 11,8 & \\
\hline \multirow[t]{3}{*}{ Faneros córneos } & & & & 0,306 \\
\hline & Claros & 65,6 & 75,8 & \\
\hline & Negro-Oscuro & 34,4 & 24,2 & \\
\hline \multicolumn{5}{|l|}{ Textura pelo } \\
\hline & Corto y fino & 100 & 100 & \\
\hline \multirow[t]{3}{*}{ Morrillo } & & & & ND \\
\hline & Ausente & 100 & 76,5 & \\
\hline & Presente & 0 & 23,5 & \\
\hline
\end{tabular}

diferentes características evaluadas.

\section{Las características presentadas en la Tabla 1 se discuten a continuación:}

La papada: Todos los animales evaluados tenían papada, presentándose discontinua en alrededor del $84 \%$ de los individuos y continua en el resto, sin que haya asociación entre machos y hembras (Tabla 1). La presencia de papada en algunas razas conforma algunas de las características que agrupan a los Bos indicus y como se desprende de lo reportado por Villasmil y Aranguren-Méndez (2005), en ganado doble propósito, en cruces hasta 82,5 \% Bos taurus no tienen papada, mientras que con mayor proporción de Bos indicus esta característica se presenta en los individuos. En este 
sentido, los resultados en este estudio contrastan con lo expresado por Barrera et al. (2006) quienes describen al Criollo Caqueteño como Bos taurus, aunque los autores indican que puede haber un bajo porcentaje de introgresión genética de Bos indicus en esta raza.

Los resultados de la papada coinciden con lo indicado por Trujillo y Floriano (2005) quienes afirman que esta característica se agrupa en continua y discontinua y con lo reportado por Sastre (2010) para el Ganado Criollo Casanareño donde el $69 \%$ de la población presenta papada discontinua. Este autor, sin embargo, encontró que 14,3\% de la población no posee papada, siendo notorio este hallazgo en el $16,7 \%$ de las hembras.

La capa: El patrón de capa predominante fue de tonalidades uniformes de un solo color e identificándose dos tonalidades principalmente (figura 2) el rojo con una mayoría (79\%) sobre el bayo $(21 \%)$. Dentro del rojo se incluyen el rojo retinto correspondiente a tres toros adultos. No se presentó relación $(\mathrm{P}<0,05)$ entre el color y el sexo. El pelo fue corto y fino en el 100\% de los animales evaluados. Esta característica coincide con lo descrito por Trujillo y Floriano (2005)

Niyas et al, 2015 asegura que animales que presentan colores más claros en su capa con pelaje pino, absorben menos calor que aquellos de colores oscuros y gruesos, lo que se presume como una adaptación evolutiva aplicable al GCC.
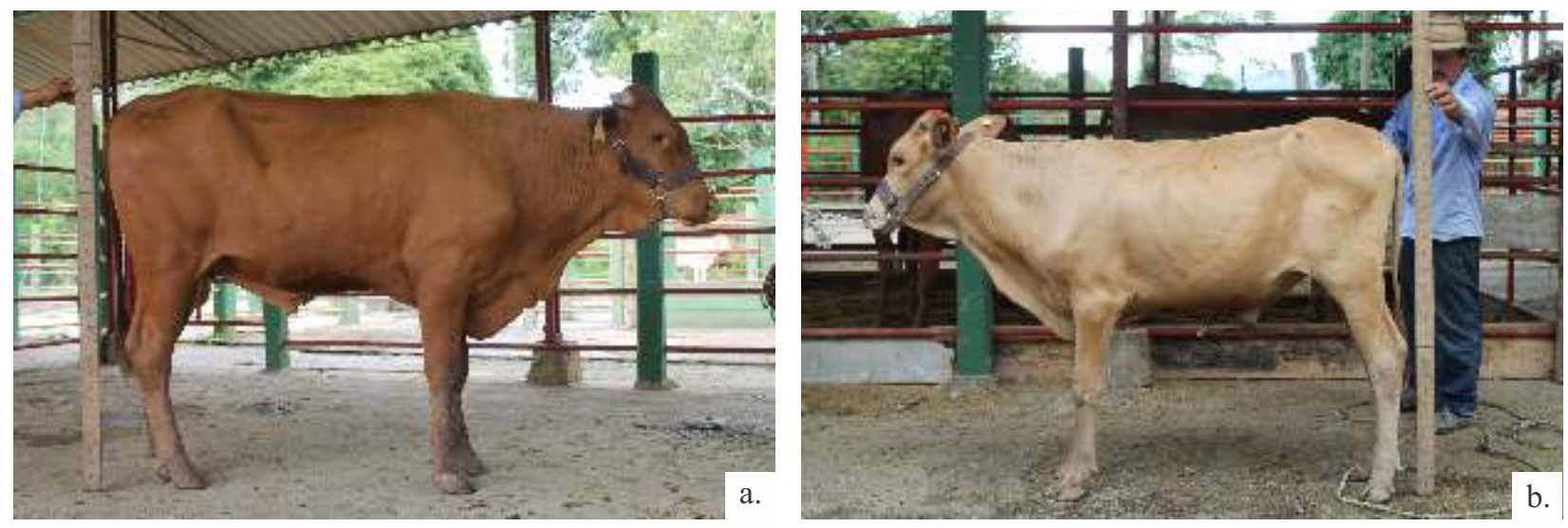

Figura 2. Patrón de tonalidades a. Rojo b. Bayo

El pliegue umbilical: E1 61\% de los animales no presentaron pliegue umbilical mientras que en el $31 \%$ esta característica estaba presente, sin que hubiera relación $(\mathrm{P}<0,05$, Tabla 1$)$ con el sexo. Los resultados coinciden con lo reportado por Sastre (2010) para el Ganado Criollo Casanareño donde el 59,5\% de la población no lo posee.

El color de las mucosas: Esta característica enmarca la coloración de la mucosa nasal, vulva y/o prepucio y color de las pezuñas y presentó tres diferentes tonalidades (sonrosadas, oscurecidas y negras). Para la prueba $x^{2}$ se sumaron las frecuencias de las oscurecidas y negras. Se observó una relación significativa $(\mathrm{P}<0,05)$ entre el color de las mucosas y el sexo de los animales. En las hembras fue más frecuente encontrar mucosas sonrosadas, mientras que en los machos el 70\% las presentó oscurecidas o negras (Tabla 1). Para el ganado Criollo Casanareño, Sastre (2010) reporta 
un $73,8 \%$ de predominio de las mucosas negras. Según Barcelo (2000), en el ganado Criollo Mallorquina, de 83 ejemplares observados, 69 tenían las mucosas pigmentadas y 14 despigmentadas.

Las Manchas: E1 87\% de los animales no presentaron manchas, sin embargo, se identificaron algunas sin relación significativa con el sexo, en hembras se observaron manchas como el meano-bragadolucero $(3,1 \%)$; lucero-meano $(1,6 \%)$; meano-bragado $(7,8 \%)$ y bragado $(1,6 \%)$. En los machos se observaron lucero en $8,8 \%$ y bragado en $2,9 \%$. Según el Comité de Ganaderos del Caquetá, la capa del GCC debe ser de un solo tono, sin embargo, se acepta que haya algunas variaciones.

En otras razas criollas como el Casanareño, se presentan diferentes tipos de manchas, similares a las del criollo Caqueteño, tales como meanas, bragadas y lucero (Sastre, 2010). El autor indica que no hay diferencias significativas en la presencia de estas manchas entre sexos para el bragado, donde el $66,7 \%$ de los machos presenta la particularidad frente a solo $11,1 \%$ de las hembras. El carácter meano presenta la misma distribución, debido en este caso, a que los animales que son meanos son bragados también. Esta relación no se guarda en el ganado Criollo Caqueteño donde se puede identificar que las características bragado y meano solo la comparten el $5 \%$ de los ejemplares diferente al $2 \%$ de los ejemplares bragados. Para el caso de lucero en el criollo casanareño, $16,7 \%$ de los machos lo presenta a diferencia de las hembras donde no hubo ningún caso (Sastre, 2010).

La Borla: Se identificaron 7 tipos de colores de borla. Sin embargo, algunas combinaciones se agruparon al rojo o al negro, pero no se realizó la prueba $x^{2}$ debido a que, como se deduce de la tabla 1 , en el $88 \%$ de los individuos predominó el rojo, sin diferencias apreciables entre hembras y machos. El porcentaje restante se repartió entre los colores negro y amarillo.

Los Faneros córneos: Se encuentran variaciones en esta característica con coloraciones oscurecidas, claras, negras y veteadas tanto en hembras como en machos. En promedio, el $71 \%$ eran claros y el resto entre negros y oscuros, sin relación con el sexo $(\mathrm{P}<0,05)$.

El Morrillo: Esta característica estuvo ausente en el 100\% de las hembras, mientras que en los machos un 23,5\% la poseía. En el estudio de Sastre (2010) ninguno de los animales criollo Casanareño presentó morrillo. La presencia de morrillo en algunos machos Criollo Caqueteño puede ser explicada, al menos en parte, por la introgresión moderada del ganado cebú indicada por Barrera etal. (2006).

\section{CONCLUSIONES}

El ganado criollo Caqueteño posee una coloración uniforme con pocas manchas y de predominio rojo, con un pelo corto y fino, características que han sido estudiadas por diferentes autores y que son destacadas como variables altamente adaptativas, lo que convierte a la raza en candidata para proyectos de reconversión ganadera.

El estudio muestra una alta homogeneidad en los bovinos Criollo Caqueteño. El tamaño de la muestra estudiada ( $75 \%$ del total de la población), proporciona confiabilidad al momento de establecer los rasgos fanerópticos más importantes. 
Estos estudios dan el primer paso para destacar la importancia de la implementación de material genético capaz de resistir el rápido cambio climático que se viene presentando en el mundo, como lo establece la FAO.

Dadas las características fenotípicas identificadas en el actual estudio para los bovinos Criollo Caqueteño, la proyectan como una raza con las condiciones adecuadas para dar rusticidad y resistencia a los cruces en la ganadería, uno de los renglones de importancia social, económica y productiva en la amazonia colombiana. Sin embargo, el bajo número de ejemplares de la raza genera en la comunidad académica una alta preocupación por la pérdida de años de adaptación.

\section{AGRADECIMIENTOS}

Al MSc, Gustavo Adolfo Celis, PhD. Jaime Enrique Velásquez R. PhD Gloria Elena Estrada y MSc, Hernan Eduardo Ocaña por su acompañamiento, asesoría y respaldo para la realización de este estudio. Y a todas las personas que trabajan en el Centro de Investigaciones Amazónica CIMAZ Macagual Cesar Augusto Estrada Gonzales, por su dedicación a la conservación de la raza de ganado Criollo Caqueteño, así como a los propietarios de los núcleos el Pensil y Villa Mery.

\section{LITERATURA CITADA}

ALDERSON, G. L. H. (1974). Genetic conservation and breed improvement: The Ark, 1:7-8p.

BARBA, C, FERNÁNDEZ, J. (2005) Paralelismo entre las razas criollas americanas y las razas autóctonas españolas. España: Archivos de zootecnia., vol. 54, no 206, p. 135-139.

BARCELÓ, T. LLITERES, B. PUIGSERVER, G.(2000) Criterios a seguir en la selección de la raza bovina Mallorquina con relación a su filogénesis. España: Archivos de zootecnia., 2000, vol. 49, no 185, p. 63-69.

ESTRADA- Cely G E (2014) Bienestar animal en la medicina veterinaria y zootecnia. Universidad de la Amazonia. Florencia, Caquetá. Colombia.

BARRERA GP, Martínez R, Torrijos R y Román F. (2006). Caracterización molecular de una población de ganado Caqueteño y su relación filogenética con razas bovinas criollas colombianas. Revista Corpoica - Ciencia y Tecnología Agropecuaria; 7:33-41. \{En línea\}. Disponible en:http://www.corpoica.org.co/SitioWeb/Archivos/Revista/4_Molecular_Characterizati on Caqueteo.pdf

BUXADÉ, Carlos (1995). Zootecnia: bases de producción animal.1 ed. Mexico D.F.: MundiPrensa,. $381 \mathrm{p}$.

EURECA (2010). European Regional Cattle Breeds. Local cattle breeds in Europe: Development of policies and strategies for self-sustaining breeds. Eureca Consortium Partners: Wageningen Academic Publishers. Disponible en: http://www.regionalcattlebreeds.eu/publications/ documents/9789086866977cattlebreeds.pdf $>$ 
FAO (2015). FOOD AND AGRICULTURE ORGANIZATION OF THE UNITED NATIONS. Breed data sheet: Cattle Breeds. Domestic Animal Diversity Information DSystem. Ciudad de Roma: Dairy Gateway. \{En línea\}. Disponible en: http://dad.fao.org/>

FAO (1990) FOOD AND AGRICULTURE ORGANIZATION OF THE UNITED NATIONS. Agricultural Investment to Promote Improved Capture and Use of Rainfall in Dryland Farming. Ciudad de Roma: Dairy Gateway. \{En línea\}. Disponible en: http://www.fao.org/3/contents/6c58 d17f-848b-52e6-ad90-db04ff37c219/v9895e00.htm>

FAO (2012a). FOOD AND AGRICULTURE ORGANIZATION OF THE UNITED NATIONS. Phenotypic characterization of animal genetic resources Ciudad de Roma: FAO, FOOD AND AGRICULTURE ORGANIZATION OF THE UNITED NATIONS., 168 p. \{En línea\}. Disponible en: http://www.fao.org/docrep/015/i2686e/i2686e00.pdf>

FAO (2012b) FOOD AND AGRICULTURE ORGANIZATION OF THE UNITED NATIONS. Report of a consultation on the definition of breed Categories: Intergovernmental technical working group on animal genetic resources for food and agriculture. Commission on genetic resources for food and agriculture. Ciudad de Roma: Dairy Gateway. 2012. 7p. \{En línea\}. Disponible en: http:/www.fao.org/docrep/meeting/026/me588e.pdf>

GUTIERREZ W; \& MARTINEZ R; \& ANZOLA H. (2003), situación de los recursos zoogeneticos en Colombia. Ed, produmedios, Bogotá DC-Colombia. \{En línea\}. Disponible en: ftp://ftp.fao.org/docrep/fao/010/a1250e/annexes/CountryReports/Colombia.pdf

HOLDRIDGE, L. R. (1978). Ecology based on life zones. (Inter-American Institute for Cooperationin Agriculture Press) San José, Costa Rica:

IDEAM (2009) Instituto de Hidrología, Meteorología y Estudios Ambientales. Datos meteorológicos del Departamento del Caquetá. Bogotá DC: Estación Limnimétrica de Montañita, Caquetá.

LAUVERGNE, J. J. (1986) Methodologie proposée pour l'etude des Ovicaprinae méditerranéens en 1986. In "J.J. Lauvergne: Traditional populations and firts standardized breeds of Ovicaprinae in the Mediterranean. Paris: INRA.,47, 77-94 p.

NIYAS PAA, CHAIDANYA K, SHAJI S, SEJIAN V, BHATTA R, ET AL. (2015) Adaptation of Livestock to Environmental Challenges. J Vet Sci Med Diagn 4:3

ORTIZ, M. (1997). Las razas autóctonas españolas y su participación en los bovinos criollos iberoamericanos. Simposium sobre utilización de Razas y Tipos Bovinos creados y desarollados en LatinoAmérica y el Caribe. Maracaibo, Venezuela: APLA., 24-28 p.

PINZON E. (1991). Historia de la ganadería bovina colombiana. Bogotá D.C: Banco Ganadero. Disponible en: <URL: https://goo.gl/9gxIhO > Vol. 8 Número 
PRIMO, A. (1992). El ganado bovino ibérico en las Américas: 500 años después. Brasil: Archivos de zootecnia., 1992. Disponible en: <URL: https://goo.gl/0T0TLr > vol.41, no 154, 421-432 p.

RODERO, S. (2002). Procedimiento normalizado de trabajo para el reconocimiento y catalogación de razas ganaderas. Córdoba, España: Sociedad Española de Zooetnología., 37 p.

R CORE TEAM. (2018). R: A language and environment for statistical computing. R Foundation for Statistical Computing, Vienna, Austria. URL https://www.R-project.org/.

SÁNCHEZ, A. y SÁNCHEZ, M. (1986). Razas Ovinas Españolas. España: Publicaciones de Extensión Agraria. Ministerio de Agricultura, Pesca y alimentación., 1986.

SAÑUDO, C. 2009. Valoración morfológica de los animales domésticos. Ministerio de Medio Ambiente y Medio Rural y Marino, Madrid, España.

SASTRE, H. J., RODERO, E., RODERO, A., HERRERA, M., \& PEÑA, F. (2009). caracterización etnológica y propuesta del estándar para la raza bovina criolla Casanare. Animal Genetic Resources, 2010, 46, 73-79. (C) Food and Agriculture Organization of the United Nations, 2010 doi:10.1017/S207863361000072X. \{En línea\}. Disponible en: http://www.fao.org/docrep/012/i1353t/i1353t10.pdf

SIERRA, I. (2001). "El concepto de raza: Evolución y realidad”. Zaragoza, España: Archivos Zootecnia., 2001. Disponible en: <URL: https://goo.gl/NxRcls> 50;192:547-564p.

TRUJILLO Y. FLORIANO L. (2005). "Características morfométricas y faneropticas de la raza". Genética Pura: Ganado Criollo Caqueteño. Florencia, Caquetá: Panamericanos., 2005

VILLASMIL W IY ARANGUREN-MÉNDEZ J A 2005 Clasificación fenotípica en vacas mestizas. En: González-Stagnaro C, Soto E, Editores. Manual de Ganadería de Doble Propósito. Ediciones Astro Data, S. A. Maracaibo, Venezuela, 75-81. 